\title{
Ronco: critérios diagnósticos e tratamento
}

Snoring: diagnostic criteria and treatment

Flávio José Magalhães da Silveira, Ricardo Luiz de Menezes Duarte

\begin{abstract}
Resumo
0 ronco é um problema importante com consequentes manifestações sociais e médicas. 0 passo inicial consiste em confirmar a presença do ronco e excluir qualquer doença respiratória noturna para que o tratamento adequado seja instituído. Este capítulo analisa o gerenciamento atual do ronco e seu tratamento.
\end{abstract}

Descritores: Ronco/diagnóstico; Ronco/cirurgia; Ronco/terapia.

\begin{abstract}
Snoring is a significant problem with social and medical manifestations. After snoring has been confirmed, other nocturnal respiratory diseases should be excluded so that the appropriate treatment can be instituted. This chapter addresses the current management of snoring and its treatment.
\end{abstract}

Keywords: Snoring/diagnosis; Snoring/surgery; Snoring/therapy.

\section{lntrodução}

0 ronco é um distúrbio comum na prática clínica. Sua prevalência varia amplamente e possui dados subestimados. No Reino Unido, a prevalência de ronco em indivíduos adultos foi estimada em 25-40\%. Outros dados sugerem que mais de 20\% dos indivíduos adultos e 50\% dos homens acima de 60 anos apresentam ronco primário. ${ }^{(1,2)}$

0 ronco surge pela produção de som pelo trato aerodigestivo durante o sono. 0 ronco primário pode ser classificado em 3 níveis (1-111), que vai de ronco ocasional (1) a ronco que pode ser ouvido do lado de fora do quarto (111). Os indivíduos com nível 111 têm uma maior probabilidade de ter SAHOS. . $^{(3,4)}$

\section{Apresentação e critérios diagnósticos}

A apresentação varia, nos extremos, desde ronco primário até SAHOS. A condição intermediária entre esses dois extremos é SRVAS. ${ }^{(4)} A$ avaliação do ronco envolve a confirmação da presença desse e a exclusão de outros distúrbios respiratórios do sono que cursam com ronco (principalmente SAHOS). A avaliação se inicia pela consulta (pelo companheiro de leito (idealmente) com dados sobre o ronco: frequência, sua relação com o decúbito e intensidade. ${ }^{(3-5)}$

0 ronco é mais comum em homens por diferenças anatômicas na região laringofaríngea e pela deposição de gordura corporal. 0 uso de sedativos/álcool relaxa a faringe e piora o ronco. 0 tabagismo piora o ronco pelo edema de mucosa e estreitamento nasofaríngeo. ${ }^{(3)}$

0 exame físico é inespecífico. Deve-se pesquisar IMC, a circunferência do pescoço, o nariz e a cavidade oral/orofaringe. ${ }^{(3)} 0$ método padrão para exclusão de distúrbio respiratório do sono é a polissonografia noturna. ${ }^{(3-5)}$ A endoscopia nasal com manobra de Müller pode ser utilizada; porém, o exame é feito com o paciente acordado e o esforço pode variar entre os pacientes, havendo variabilidade entre os observadores. ${ }^{(3,6)}$ A nasoendoscopia durante o sono avalia o nível de colapso enquanto o paciente está sedado, mas a redução no diâmetro faríngeo pode ser causado pela sedação.(3,6) Exames como cefalometria, TC e ressonância magnética podem também ser utilizados. ${ }^{(3)}$

\section{Tratamento}

0 tratamento consiste em: a) intervenção conservadora: perda de peso, correção do decúbito, restrição no uso de sedativos/álcool e tratamento da obstrução nasal e do tabagismo; b) intervenção não cirúrgica: dispositivo intraoral e CPAP; e c) cirurgia: nasal, do palato e bariátrica. ${ }^{(3,4,6)}$

Perder peso é uma medida eficaz a curto prazo, mas difícil a longo prazo.(1) Há mais estudos com dispositivo intraoral em SAHOS do que em ronco primário: sua adesão gira em torno de 50-75\%. 0 dispositivo intraoral com 
avanço mandibular reduz a frequência, duração e intensidade do ronco. 0 dispositivo intraoral com retenção de língua pode ser utilizado naqueles com dentição insuficiente e que não podem utilizar o avanço mandibular. ${ }^{(6)} 0$ uso de CPAP é a forma mais eficaz para o tratamento de ronco primário, mas o desconforto causado pelo aparelho reduz a adesão. ${ }^{(3)}$

A cirurgia nasal pode beneficiar poucos pacientes. A uvulopalatofaringoplastia possui eficácia a longo prazo menor que 50\% A uvulopalatoplastia por laser possui resultados a longo prazo de $45-75 \% .{ }^{(3)}$ A cirurgia intrapalatal tem baixa morbidade e possui resultados a curto prazo de $30-60 \%{ }^{(3,6)}$

\section{Referências}

1. Stradling JR, Crosby JH. Predictors and prevalence of obstructive sleep apnoea and snoring in 1001 middle aged men. Thorax. 1991;46(2):85-90.

2. Lugaresi E, Cirignotta F, Coccagna G, Piana C. Some epidemiological data on snoring and cardiocirculatory disturbances. Sleep. 1980;3(3-4):221-4.

3. Counter P, Wilson JA. The management of simple snoring. Sleep Med Rev. 2004;8(6):433-41.

4. Trotter MI, D'Souza AR, Morgan DW. Simple snoring: current practice. J Laryngol Otol. 2003;117(3):164-8.

5. Bloom JW, Kaltenborn WT, Quan SF. Risk factors in a general population for snoring. Importance of cigarette smoking and obesity. Chest. 1988;93(4):678-83.

6. McGown AD, Makker HK, Battagel JM, L'Estrange PR, Grant HR, Spiro SG. Long-term use of mandibular advancement splints for snoring and obstructive sleep apnoea: a questionnaire survey. Eur Respir J. 2001;17(3):462-6.

\section{Sobre os autores}

Flávio José Magalhães da Silveira

Diretor Médico. SLEEP - Laboratório de Estudo dos Distúrbios do Sono, Centro Médico BarraShopping, Rio de Janeiro (RJ) Brasil.

\section{Ricardo Luiz de Menezes Duarte}

Professor Convidado da Cadeira Cardiopulmonar. Universidade Federal do Estado do Rio de Janeiro - UNIRIO - e Diretor Médico. SLEEP - Laboratório de Estudo dos Distúrbios do Sono, Centro Médico BarraShopping, Rio de Janeiro (RJ) Brasil.

E-mail para contato: flaviomagalhaes1@gmail.com (F. Silveira) 